\title{
Polymers from 1,2-Disubstituted Ethylenic Monomers VI. Monomer-Isomerization Radical Polymerization of Diethyl Maleate
}

\author{
Naoyuki TOYODA, Masatoshi YoSHIDA, and Takayuki OTSU \\ Department of Applied Chemistry, Faculty of Engineering, \\ Osaka City University, Sugimoto, Sumiyoshi-ku, Osaka 558, Japan
}

(Received September 16, 1982)

\begin{abstract}
In contrast to diethyl fumarate (DEF), diethyl maleate (DEM) did not homopolymerize with 2,2'-azobisisobutyronitrile (AIBN), but was found to undergo radical polymerization in the presence of amines such as morpholine (Mor). From the results of radical polymerization of DEF with AIBN and isomerization of DEM with Mor, the radical polymerization of DEM in the presence of both AIBN and Mor is considered to proceed via a new monomer-isomerization radical polymerization mechanism, i.e., DEM is isomerized first to DEF which is then homopolymerized. The most efficient isomerization catalyst was Mor among the amines examined. The apparent activation energies for the monomer-isomerization radical polymerization of DEM was calculated to be $91.9 \mathrm{~kJ} \mathrm{~mol}^{-1}$ and that for this isomerization was also obtained as $24.7 \mathrm{~kJ} \mathrm{~mol}^{-1}$. Moreover, Mor also acted as a retarder of radical polymerization of DEF. The composition relation of the copolymer to the feed monomer mixture in the copolymerizations of DEM with styrene, isobutyl vinyl ether, and acrylonitrile in the presence of Mor was found to be similar to that of the respective copolymerizations of DEF.
\end{abstract}

KEY WORDS Radical Polymerization / Radical Copolymerization / Monomer-Isomerization Radical Polymerization / Diethyl Maleate / Diethyl Fumarate /

It is known that 1,2-disubstituted ethylenic monomers except vinylene carbonate ${ }^{1}$ and maleimide derivatives ${ }^{2}$ homopolymerize only to a very small extent in the presence of initiator, because of the steric hindrance of the substituents. However, 1,2-disubstituted olefins such as 2-butene, 2-pentene, and 4-phenyl-2-butene were found to polymerize with the Ziegler-Natta catalyst to give high molecular weight homopolymers consisting of the respective 1-olefin monomer unit. ${ }^{3}$ In these polymerizations, 2-olefins isomerized first to the corresponding 1-olefins which then homopolymerized, and we termed these polymerizations "monomerisomerization polymerization" for which the data obtained from our laboratory have been reported in detail.

Recently, various dialkyl fumarates, trans-1,2disubstituted monomers, were found to undergo homopolymerization in the presence of a radical initiator to give relatively high molecular weight homopolymers, but the respective cis isomers, i.e., dialkyl maleates, did not give any homopolymers under similar conditions. ${ }^{4-8}$ However, when amines such as morpholine (Mor) were added to these systems, dialkyl maleates were also found to polymerize. ${ }^{4,6}$

In previous papers, the results of the radical homopolymerization of dimethyl fumarate ${ }^{7}$ and dimethyl maleate ${ }^{8}$ in either the presence or absence of amines were reported. In order to clarify further the detailed mechanism of the monomerisomerization radical polymerization of dialkyl maleates, the radical polymerization of diethyl maleate (DEM) was undertaken in this study. The results obtained are described in relation to the radical polymerization of diethyl fumarate (DEF).

\section{EXPERIMENTAL}

\section{Materials}

Commercial DEM and DEF were used after fractional distillation under reduced pressure. The 
purities of both monomers were above $99 \%$. Styrene (St), acrylonitrile (AN), and isobutyl vinyl ether (IBVE) used in the copolymerizations were purified by ordinary methods, and distilled under reduced pressure in a nitrogen atmosphere before use.

2,2'-Azobisisobutyronitrile (AIBN), radical initiator, was recrystallized from methanol, and dried in vacuum. Morpholine (Mor), piperidine, $n$ propylamine, di- $n$-propylamine, tri- $n$-propylamine, aniline, $p$-nitroaniline, $p$-anisidine, $p$-toluidine, and pyridine, used as the isomerization catalysts were distilled before use. Benzene and the other reagents were used after purification by ordinary methods.

\section{Polymerization Procedure}

The polymerizations of DEM and DEF were carried out in a degassed glass tube with an AIBN initiator in both the presence and absence of the amine as isomerization catalyst in benzene at $50-$ $90^{\circ} \mathrm{C}$. After polymerization for a given time, the content of the tube was poured into a large amount of $n$-hexane to isolate the polymer. The resulting polymer was then reprecipitated from a system of benzene and $n$-hexane, and dried in vacuum at room temperature. Similar procedures were applied to radical copolymerizations performed to low conversions $(\mathrm{ca} .10 \%)$. The composition of the copolymers was analyzed by elemental analysis, and then calculated from their carbon or nitrogen content.

\section{Isomerization of DEM to DEF}

The isomerizations of DEM to DEF were carried out in benzene in the presence of Mor. A required amount of the reaction mixture was taken out from the system at regular time intervals, and the ratios of DEM to DEF were determined by gas chromatography (column: PEG 6000) or ${ }^{1} \mathrm{H}$ NMR spectrometry in carbon tetrachloride, and toluene used as a reference.

\section{Characterization of the Polymer}

The structure of the polymers was checked by IR, ${ }^{1} \mathrm{H}$ NMR, and ${ }^{13} \mathrm{C}$ NMR spectra. The intrinsic viscosity $([\eta])$ was determined in benzene at $30^{\circ} \mathrm{C}$. The softening temperature was measured with a micromelting point apparatus. A differential scanning calorimeter (DSC) was used for the determination of the glass transition temperature $\left(T_{\mathrm{g}}\right)$ at a heating rate of $10^{\circ} \mathrm{C} \mathrm{min}^{-1}$ in a helium atmosphere. Thermal degradation studies were carried out in a nitrogen atmosphere at a heating rate of $10^{\circ} \mathrm{C}$ $\min ^{-1}$, using a Shimadzu TGC-20 thermobalance.

\section{RESULTS AND DISCUSSION}

\section{Radical Polymerization of DEM and DEF in the} Presence or Absence of Some Amines

The results of the radical polymerization of DEM with AIBN in the presence and absence of various amines are shown in Table I along with some results of DEF.

As can be seen from this table, the polymerization of DEM did not take place in the absence of the amines but did so for DEF. However, when a small amount of an amine is added to this system, the polymerization of DEM is induced. Among the amines examined, primary and secondary aliphatic amines are effective for isomerization. Mor shows the most efficient activity for DEM, but it also acts as a retarder for the polymerization of DEF (see

Table I. Isomerization and polymerization of $\mathrm{DEM}$ in bulk at $60^{\circ} \mathrm{C}^{\mathrm{a}}$

\begin{tabular}{|c|c|c|}
\hline \multirow{2}{*}{ Amine } & \multirow{2}{*}{$\begin{array}{c}\begin{array}{c}\text { Isomerization } \\
\text { from }\end{array} \\
\text { DEM to DEF } \\
\%\end{array}$} & \multirow{2}{*}{$\frac{\text { Polymer yield }}{\%}$} \\
\hline & & \\
\hline None & 0 & 0 \\
\hline Propylamine & 100 & 0.7 \\
\hline Dipropylamine & 100 & 2.9 \\
\hline Tripropylamine & 0 & 0 \\
\hline Morpholine & 100 & $9.8(0.04)$ \\
\hline Piperidine & 100 & 4.8 \\
\hline Aniline & 0 & 0 \\
\hline$p$-Nitroaniline & 0 & 0 \\
\hline$p$-Toluidine & 0 & 0 \\
\hline$p$-Anisidine & 0 & 0 \\
\hline Pyridine & 0 & 0 \\
\hline None $(\mathrm{DEF})^{\mathrm{c}}$ & $(0)^{d}$ & $13.2(0.2)$ \\
\hline None $(\mathrm{DEF})^{\mathrm{e}}$ & $(0)^{d}$ & 10.0 \\
\hline
\end{tabular}

a Experimental conditions: $[\mathrm{DEM}] /[$ Amine $]=26.6$; $[\mathrm{AIBN}]=20 \mathrm{mmol}^{-1}$; time, $16 \mathrm{~h}$.

b Values in parenthese indicate those of $[\eta]$ in $\mathrm{dlg}^{-1}$.

c Indicates the results obtained from DEF used as a starting monomer in the absence of morpholine.

${ }^{d}$ DEF did not isomerize to DEM.

e Indicates the results obtained from DEF in the presence of morpholine. 
Figure 4).

The aromatic amines are not effective for both isomerization and polymerization. Also, tertiary amines do not serve as isomerization catalysts, because of the absence of hydrogen on their nitrogen atoms and increased steric requirement by three alkyl groups. The unreacted monomer recovered after the polymerization of DEM in the presence of effective amines was found to be completely the DEF monomer, indicating that the isomerization of DEM to DEF took place. Under these conditions, however, the reverse isomerization from DEF to DEM was not observed. In the absence of Mor, no isomerizations from DEF to DEM and from DEM to DEF were found to occur.

As is described in a later section, the resulting polymer obtained from DEM was quite identical to that from DEF which was confirmed to consist of the poly(ethoxycarbonylmethylene) structure as a result of a vinylene polymerization. Thus, it is evident that the polymerization of DEM is induced if the starting DEM isomerizes to DEF, i.e., DEM isomerizes first to DEF with the effective amine, and then undergoes a radical homopolymerization. Recently, we termed this polymerization "monomer-isomerization radical polymerization." 4 Similar polymerizations were also found to occur for various dialkyl maleates. ${ }^{4,6,8}$

Kinetic Study of Monomer-Isomerization Radical Polymerization of DEM in the Presence of Mor

The time-conversion relations for bulk polymerization of DEM in the presence of both AIBN and Mor at $50-90^{\circ} \mathrm{C}$ are shown in Figure 1 .

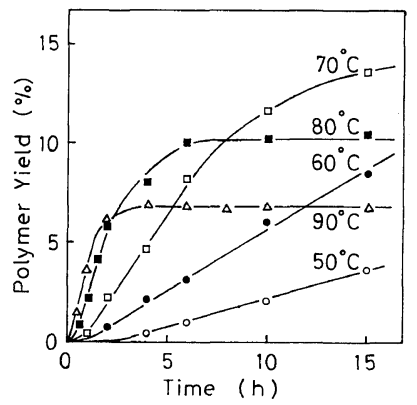

Figure 1. Time-conversion relations for radical polymerization of DEM in the presence of Mor.: $[\mathrm{DEM}]=6.13 \mathrm{moll}^{-1}$; $[\mathrm{AIBN}]=20 \mathrm{mmoll}^{-1}$; [Mor] $=$ $0.11 \mathrm{moll}^{-1}$.
From this figure, a short induction period is seen in the initial stage of polymerization for the temperature examined, and the following polymerization proceeds linearly up to $\mathrm{ca}$. $10 \%$ conversion at temperatures from 50 to $70^{\circ} \mathrm{C}$. At 80 and $90^{\circ} \mathrm{C}$, the rates of polymerization gradually decrease with the time and approach a constant conversion. This seems to result from a dead-end polymerization, ${ }^{9}$ because of the short half-life of AIBN at these temperatures.

The observed induction periods are shortened with increasing polymerization temperature, indicating that they apparently correspond to the time necessary to isomerize from DEM to DEF as will be shown later.

From the initial slopes of the time-conversion relations of Figure 1, the rates of polymerization $\left(R_{\mathrm{p}}\right)$ were determined. Arrhenius plots of $\log R_{\mathrm{p}}$ with the reciprocals of the absolute polymerization temperatures are shown in Figure 2, from which the activation energy for this monomer-isomerization radical polymerization of DEM was calculated to be $91.9 \mathrm{~kJ} \mathrm{~mol}^{-1}$. This value is somewhat lower than that $\left(95.9 \mathrm{~kJ} \mathrm{~mol}^{-1}\right)^{8}$ obtained for dimethyl maleate, possibly as a results of the temperature range $\left(100-130^{\circ} \mathrm{C}\right)$ and initiator (di-tert-butyl peroxide) used.

\section{Isomerization of DEM to DEF with Mor}

To clarify the isomerization of DEM to DEF, reactions of DEM with Mor were carried out in carbon tetrachloride at temperatures between $25^{\circ}$ and $70^{\circ} \mathrm{C}$, and analyzed by NMR spectra. The amounts of DEF isomerized from DEM were plotted against reaction time. The results are shown in

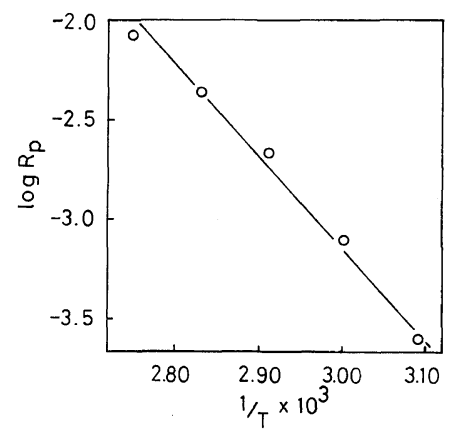

Figure 2. Arrhenius plot for monomer-isomerization radical polymerization of DEM. 


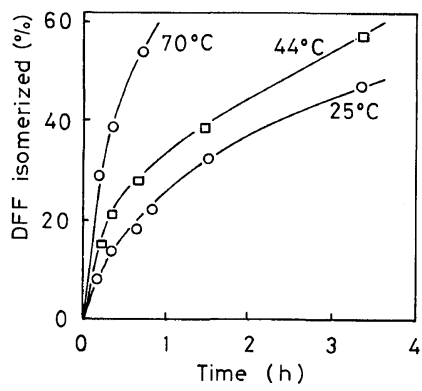

Figure 3. Isomerization of DEM with Mor to DEF in carbon tetrachloride: $[\mathrm{DEM}]=3.10 \mathrm{moll}^{-1}$; $[\mathrm{Mor}]=62$ $\mathrm{mmoll}^{-1}$.

Table II. Pseudo-first order reaction rate constants $(k)$ and activation energy

$(E)$ for isomerization of DEM with Mor ${ }^{\mathrm{a}}$

\begin{tabular}{|c|c|c|}
\hline Temperature & $k \times 10^{4}$ & $E$ \\
\hline${ }^{\circ} \mathrm{C}$ & $1 \mathrm{~s}^{-1}$ & $\mathrm{~kJ} \mathrm{~mol}^{-1}$ \\
\hline 25 & 0.96 & \\
\hline 44 & 1.73 & 24.7 \\
\hline 70 & 3.55 & \\
\hline
\end{tabular}

Figure 3, from which the isomerization of DEM to DEF can be seen to take place rapidly even at relatively low temperatures.

From the initial slopes of the observed relations, the pseudo-first order rate constants $(k)$ of isomerizations were calculated as shown in Table II. From: the Arrhenius plots of $\log k$ against the reciprocal absolute temperatures, the activation energy for this isomerization was calculated to be $24.7 \mathrm{~kJ} \mathrm{~mol}^{-1}$. Although the isomerization of DEM to the polymerizable DEF occurs with Mor, a short induction period seems to appear early in the polymerization (see Figure 1).

The isomerization from maleates is known generally to be catalyzed by primary or secondary amines (Table I), and to proceed via the following three reaction steps ${ }^{10-12}$ : a) reversible 1,4 -addition of the amine to DEM, b) rotation of the carboncarbon bond of the intermediate, and c) elimination of the amine. Therefore, when the Mor, a secondary amine, was added, the isomerization of DEM to

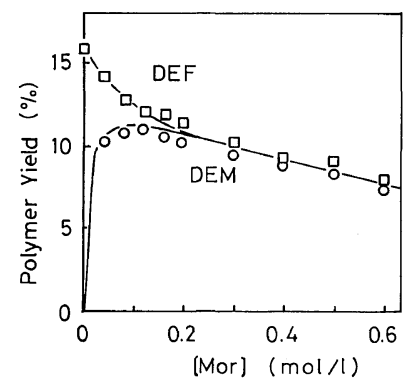

Figure 4. Relationship between polymer yield and Mor concentration in the polymerization of DEF ( $\square$ ) and monomer-isomerization polymerization of DEM (O) in benzene at $60^{\circ} \mathrm{C}$ for $20 \mathrm{~h}:[\mathrm{AIBN}]=20 \mathrm{mmoll}^{-1}$; $[\mathrm{DEF}]=[\mathrm{DEM}]=5.1 \mathrm{moll}^{-1}$.

DEF seems to proceed in a similar way.

Effect of Mor on Isomerization and Polymerization Since the monomer-isomerization radical polymerization of DEM involves both isomerization to $\mathrm{DEF}$ and the radical polymerization of $\mathrm{DEF}$, it is important to investigate the effect of Mor on the radical polymerization of DEM and DEF in the presence of AIBN at $60^{\circ} \mathrm{C}$. The results are shown in Figure 4.

When the concentration of Mor increases in the radical polymerization of $\mathrm{DEF}$, the polymer yields decrease gradually, indicating that Mor acts as a retarder of the DEF polymerization. Since amines act as a typical retarders for radical polymerization, in the present case the retardation seems to result from an abstraction by the polymer radical of DEF a hydrogen atom from Mor, and the nitrogencentered radical thus formed does not participate in reinitiation with the DEF monomer.

When using DEM as starting monomer, on adding a small amount of Mor, the polymer formation is observed, but the polymer yield decreases with increasing Mor concentration. These findings from both DEM and DEF are considered to fit an identical curve (the curve drawn in Figure 1 reported in the previous communication ${ }^{4}$ is not correct and should be changed to that of Figure 4 of this paper). Therefore, Mor acts as both a catalyst for the isomerization of DEM to DEF and a retarder of the radical polymerization of DEF as stated above. 


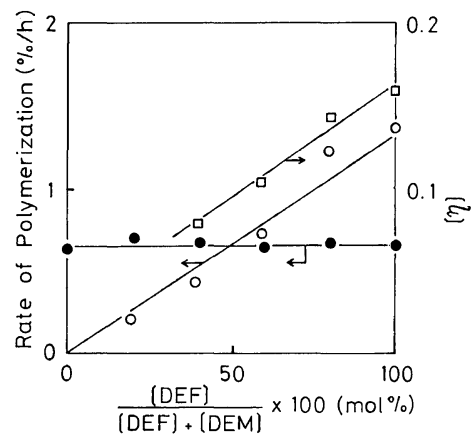

Figure 5. Effect of DEM on radical polymerization of $\mathrm{DEF}$ in bulk at $60^{\circ} \mathrm{C}:[\mathrm{AIBN}]=20 \mathrm{mmoll}^{-1}$; (O), $[\mathrm{Mor}]=0 ;(\bigcirc),[\mathrm{Mor}]=0.11 \mathrm{moll}^{-1}$.

\section{Effect of DEM on Radical Polymerization of DEF}

In the monomer-isomerization polymerization of 2-butene by Ziegler-Natta catalyst to give poly(1butene), 2-butene is known to serve as only an inert diluent for the polymerization of 1-butene. To investigate whether the DEM can also participate in the radical polymerization of DEF in this case, polymerizations of DEF were carried out at various concentrations of DEM in the presence and absence of Mor at $60^{\circ} \mathrm{C}$. The results are shown in Figure 5.

In the absence of Mor, the polymer yields decrease linearly with increasing feed DEM concentration, probably indicating that DEM acts as inert diluent in the polymerization of DEF, i.e., no copolymerization of DEF with DEM take place. In fact, the ratio of reactivity of DEF to DEM toward the polystyryl radical was calculated from the copolymerization parameters (see Table III) to be 27.5, i.e., DEF is 27.5 times more reactive than DEM.

In the presence of Mor, however, the polymer yields are independent of feed DEM concentration, and also lower than those observed from the bulk polymerization of DEF. These results seem to coincide with the fact that Mor acts as both an effective isomerization catalyst and a retarder of the radical polymerization of DEF as stated above.

\section{Radical Copolymerizations in the Presence or Absence of Mor}

In a previous paper, ${ }^{6}$ the relationship between feed monomer composition and copolymer composition for the copolymerization of St with DEM in the absence of Mor was different from that of St with DEF, but in the presence of Mor, this re-
Table III. Monomer reactivity ratios of copolymerization of St, IBVE, and $\operatorname{AN}\left(M_{1}\right)$ with $\operatorname{DEF}$ or $\operatorname{DEM}\left(\mathbf{M}_{2}\right)$

\begin{tabular}{|c|c|c|c|c|}
\hline $\mathrm{M}_{1}$ & $\mathrm{M}_{2}$ & $\frac{[\text { Mor }]}{\mathrm{moll}^{-1}}$ & $r_{1}$ & $r_{2}$ \\
\hline St & DEM & 0 & 8.0 & 0.07 \\
\hline $\mathrm{St}$ & DEM & 0.28 & 0.35 & 0.02 \\
\hline St & DEF & 0 & 0.29 & 0.02 \\
\hline IBVE & DEM & 0 & 0.0 & 0.17 \\
\hline IBVE & DEM & 0.28 & 0.0 & 0.43 \\
\hline IBVE & DEF & 0 & 0.0 & 0.52 \\
\hline $\mathrm{AN}$ & DEM & 0 & 20 & 0.00 \\
\hline AN & DEM & 0.28 & 9 & 0.20 \\
\hline AN & DEF & 0 & 10 & 0.10 \\
\hline
\end{tabular}

lationship for both systems was found to be basically the same. The same was also found for the relationship between feed monomer composition and the copolymerization rate.

To further confirm this point, the copolymerizations of IBVE with DEF and DEM initiated by AIBN were carried out in the presence and absence of Mor at $60^{\circ} \mathrm{C}$. When Mor was added to the IBVE-DEM system, the observed relation between copolymer composition and the feed monomer composition was found to be similar to that of the IBVE-DEF system. However, in the absence of Mor, the relation for the IBVE-DEM system is different from that obtained for the IBVE-DEF system.

Similar results were also observed for the copolymerizations of AN with DEF or DEM in the presence and absence of Mor. Moreover, the relation between copolymerization rate and feed monomer composition for both copolymerizations with DEM in the presence of Mor was found to be similar to that of respective copolymerizations with DEF in the absence of Mor.

The apparent monomer reactivity ratios determined for these copolymerizations are summarized in Table III. From this table, it should be noted that in the absence of Mor, DEM is much less reactive than DEF toward attack of polymer radicals, but in the presence of Mor, it shows basically the same reactivity toward DEF. Moreover, the results obtained from the "monomer-isomerization radical copolymerization" of maleate monomers are explained satisfactorily from the monomer- 


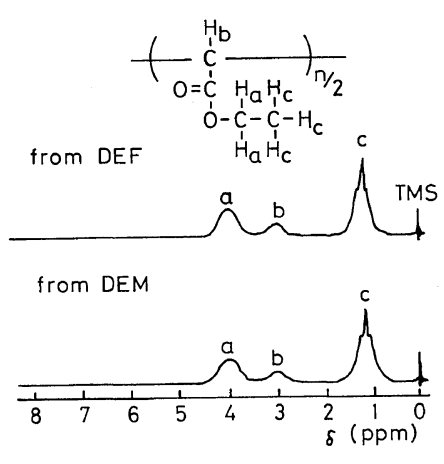

Figure 6. ${ }^{1} \mathrm{H}$ NMR spectra of the polymers obtained from DEF and DEM.

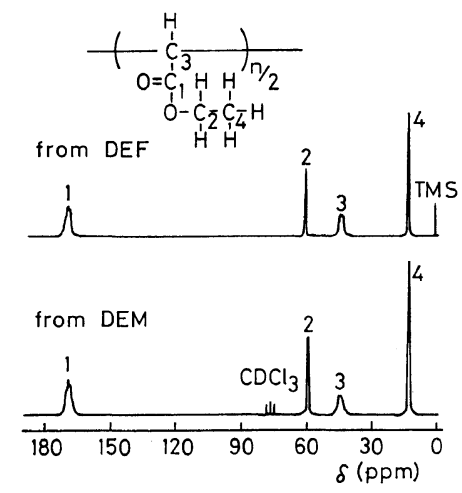

Figure 7. ${ }^{13} \mathrm{C}$ NMR spectra of the polymers obtained from DEF and DEM.

isomerization radical polymerization mechanism of DEM as described above.

\section{Characterization of Polymers Obtained from DEM or DEF}

The IR spectrum of the polymer obtained from $\mathrm{DEM}$ was quite similar to that from $\mathrm{DEF}$, i.e., the absorption bands at $1630-1640$ and $980 \mathrm{~cm}^{-1}$ due to the double bond of the monomers disappeared in both polymers. The ${ }^{1} \mathrm{H}$ and ${ }^{13} \mathrm{C}$ NMR spectra of the polymers obtained from DEF and DEM are shown in Figures 6 and 7, respectively. The spectra of these polymers are quite identical. That is, in ${ }^{1} \mathrm{H}$ NMR, the observed broad absorptions at 1.3, 3.2, and 4.1 ppm are assigned to the protons of methyl, methine, and methylene, respectively. From ${ }^{13} \mathrm{C}$ NMR, the absorptions found at $14,45,60.5$, and $170 \mathrm{ppm}$ are also due to the carbons of methyl, methine, methylene, and carbonyl, respectively. Since these bands are also found in the DEF polymers, it is thus clear that the polymer from DEM is produced through a vinylene polymerization of DEF.

The polymers obtained from both DEM and DEF were colorless powders soluble in most organic solvents such as benzene, acetone, methanol, tetrahydrofuran, and dimethylformamide except $n$ hexane and water. The softening and glass transition temperatures of the polymer from DEM with low molecular weight $\left([\eta]=0.04 \mathrm{dl} \mathrm{g}^{-1}\right)$ were found to be $78-80^{\circ} \mathrm{C}$ and $65^{\circ} \mathrm{C}$, respectively, being somewhat higher than those corresponding $\left(62-72^{\circ} \mathrm{C}\right.$ and $\left.40^{\circ} \mathrm{C}\right)^{13}$ to the polymer from DEF with a relatively high molecular weight $\left([\eta]=0.20 \mathrm{dl} \mathrm{g}^{-1}\right)$.

The TGA thermogram of the polymer obtained from DEM was also similar to that obtained by $\mathrm{DEF},{ }^{5}$ i.e., the initial and maximum degradation temperatures were determined as $248^{\circ}$ and $378^{\circ} \mathrm{C}$, respectively. These results provide some supports that the polymers obtained from both DEM and DEF have identical structures and that DEM is polymerized through a monomer-isomerization radical polymerization mechanism.

\section{REFERENCES}

1. M. S. Newman and R. W. Addor, J. Am. Chem. Soc., 75, 1235 (1935).

2. P. O. Tawny, J. Org. Chem., 26, 15 (1961).

3. T. Otsu, A. Shimizu, and M. Imoto, J. Polym. Sci., $A-1,4,1579$ (1966); see ref 4.

4. T. Otsu and N. Toyoda, Makromol. Chem., Rapid Commun., 2, 79 (1981).

5. T. Otsu, O. Ito, and N. Toyoda, Makromol. Chem., Rapid Commun., 2, 725 (1981).

6. T. Otsu, O. Ito, and N. Toyoda, Makromol. Chem., Rapid Commun., 2, 729 (1981).

7. T. Otsu, O. Ito, N. Toyoda, and S. Mori, Mem. Fac. Eng., Osaka City Univ., 22, 129 (1981).

8. T. Otsu, O. Ito, and N. Toyoda, J. Macromol. Sci.Chem., in press.

9. A. V. Tobolsky, J. Am. Chem. Soc., 80, 5927 (1958).

10. G. R. Clemo and S. B. Graham, J. Chem. Soc., 213, (1930).

11. S. Inoue, S. Ohashi, and Y. Unno, Polym. J., 3, 611 (1972).

12. Th. Wagner-Jauregy and Q. Ahmed, Helv. Chim. Acta, 57, 1871 (1974).

13. T. Otsu, T. Ishikawa, and N. Toyoda, Polym. Prepr. Jpn., 28, 28 (1979); to be published. 